\title{
COMMENT
}

\section{When is a potential new neuroprotective treatment ready for translation?}

\author{
Joanne O. Davidson ${ }^{1}$, Laura Bennet ${ }^{1}$, Sidhartha $\operatorname{Tan}^{2}$ and Alistair J. Gunn (iD ${ }^{1}$ \\ Pediatric Research (2020) 87:620-621; https://doi.org/10.1038/s41390-019-0673-4
}

Now that therapeutic hypothermia for neonatal encephalopathy (NE) has successfully translated to routine care, ${ }^{1}$ the key challenge is to find adjunct therapies that can safely further improve outcomes. ${ }^{2}$ Selection is complicated by the curse of choice, as over 1000 different interventions are reported to show neuroprotection in various settings. In the present issue of Pediatric Research, Favié et al. $^{3}$ report a two-phase trial to establish the pharmacokinetics and short-term safety of a potential adjuvant, an inhibitor of neuronal and inducible nitric oxide synthase (NOS), 2iminobiotin. The second phase was needed as the initial dose regime was an approximation derived from piglet studies undertaken during normothermia. ${ }^{4}$ Species differences, global hepatic and renal injury associated with $\mathrm{NE}$, and of course therapeutic hypothermia itself, all can, and commonly do, substantially affect drug clearance. This is a nice example of one of the steps needed for clinical translation.

The authors highlight that in the piglet study 2-iminobiotin was given for just $24 \mathrm{~h}$, whereas in this dose finding study, they chose to give it for $48 \mathrm{~h}^{3}$ based on limited animal data. There is no human evidence for the duration of upregulation of NOS in NE or for the optimal duration of therapy in animal studies. They conclude that human studies are now needed to establish the optimal duration of therapy. This is an important time to reflect on what strategy we should use to translate promising interventions and whether it is important to establish pharmacodynamics first. Some of the issues are highly specific for neonatal neuroprotection, while others are common across many areas of pediatric research.

\section{HOW "STRONG" IS THE EVIDENCE FOR NEUROPROTECTION?}

As recently reviewed, there is moderately strong evidence for 2iminobiotin, in more than one species. ${ }^{5}$ One dose ranging study in the piglet demonstrated functional and histological benefits, ${ }^{4}$ when treatment was started immediately after hypoxia-ischemia ( $\mathrm{HI})$. In other studies of this agent, it was given either before or shortly after $\mathrm{HI}^{5}$ More generally, there is only limited evidence for delayed treatment with any NOS inhibitor. This is a substantial limitation, since it remains a formidable challenge to start new interventions shortly after birth. For example, in the foundation randomized controlled trials (RCTs) of therapeutic hypothermia, infants were typically randomized at a mean of 4.0-5.2 h after birth. ${ }^{6}$ Of concern, some recent trials have reported even later initiation of treatment. For example, in a recent phase two study of an injectable adjunct treatment, erythropoietin, the first dose was given at a mean of 16.5 (5.9) h. ${ }^{7}$ Thus, unless it is clear how much delay is acceptable, it would be very easy for excessive delay to confound the results of an RCT.

IS IT NEUROPROTECTIVE WITH THERAPEUTIC HYPOTHERMIA? The next issue is that the animal studies of 2-iminobiotin were undertaken during normothermia. Therapeutic hypothermia suppresses multiple extracellular and intracellular processes leading to programmed cell death. ${ }^{8}$ Its effect on adjunct therapies is complex. It can delay the progression of cell death, and so in some studies, increased the window of opportunity for other interventions. Conversely, it suppresses many potential target processes, and so could reduce the effectiveness of adjunct interventions. Indeed, a recent study in P9 mice found that combination therapy with hypothermia and stem cell therapy was less effective than either alone. ${ }^{9}$

Of immediate relevance to the present study, in anesthetized piglets, hypothermia for $5 \mathrm{~h}$ significantly attenuated the posthypoxic increase in extracellular NO. ${ }^{10}$ Similarly, hypothermia suppressed inducible NOS expression $12 \mathrm{~h}$ after $\mathrm{HI}$ in newborn piglets, ${ }^{11}$ and $8 \mathrm{~h}$ after cardiac arrest in adult pigs. ${ }^{12}$ This raises the plausible scenario that the effects of 2-iminobiotin may be less than additive or even non-additive during co-treatment with hypothermia. Further, it is unclear whether or to what extent 2-iminobiotin could also inhibit beneficial epithelial NOS activity in humans, and whether its treatment effects are sex specific, as suggested by rodent studies. ${ }^{5}$ Thus, we submit that formal studies of co-treatment with a clinical hypothermia regime to determine the optimal dose and duration in the context of realistic delays before initiating treatment are essential before committing the substantial resources needed for RCTs powered to demonstrate neuroprotection and to compare treatment regimes.

\section{CONCLUSION}

There is obviously no one correct approach to translation. Rather, for each potential intervention we need to carefully consider the pragmatic balance between different types of risk. On the one hand, there is a risk of unnecessarily delaying progress on a highly promising candidate if we need to wait for additional animal data. This risk should be balanced against the risk of wasting clinical resources on an agent whose effects are not additive with

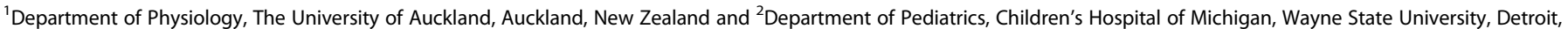
MI, USA

Correspondence: Alistair J. Gunn (aj.gunn@auckland.ac.nz)

Received: 19 October 2019 Revised: 24 October 2019 Accepted: 30 October 2019

Published online: 9 November 2019 
hypothermia due to overlapping mechanisms of action, especially if the drug target is unclear. Moreover, if clinical trials are started before there is strong information on the most effective trial protocol, and the likely magnitude of effect, there is a substantial risk of inconclusive results and lack of progress from incomplete information, leading to prematurely abandoning a beneficial intervention.

The animal studies of therapeutic hypothermia translated strikingly well in to clinical practice. Partly, this reflected a very broad evidence base from multiple competing groups, experimental paradigms, and species. Partly, it also reflected highly focused work from multiple investigators to establish the effective window of opportunity, optimal dose range, and duration. ${ }^{1}$ Subsequent animal studies and a very large RCT undertaken in parallel have shown highly similar results, refining our knowledge of the optimal regime for therapeutic hypothermia, and supporting the translational value of well-conducted animal studies. ${ }^{13,14}$ It is reasonable to note that many of these questions have not been answered for 2-iminobiotin.

The challenges inherent in trying to answer clinical questions with RCTs must not be underestimated. It is easy to forget that we did not have statistically significant evidence for therapeutic hypothermia until the meta-analysis of the first three RCTs. ${ }^{15}$ In practice, many regulatory authorities did not support routine use of therapeutic hypothermia until the outcomes from over 1100 randomized infants were available. ${ }^{6}$ In the era of therapeutic hypothermia, trial power will be less because of the reduced event rate, and thus extremely large trials will be needed to resolve multiple questions and show effect, even for a highly additive adjunct therapy. Given these issues, there is a danger that clinical studies would not be sufficiently well powered to determine the window of opportunity or optimal duration (or dose) for this or any other therapeutic target. If an early study suggested no effect by chance or because the first treatment protocol was not optimal, it would be extremely difficult to support ongoing studies.

In conclusion, we strongly recommend that further key animal studies using well-controlled, translational models are undertaken to determine the optimal dose and duration of co-treatment with therapeutic hypothermia, in the setting of clinically plausible delays after $\mathrm{HI}$, before undertaking RCTs with the power to reliably test neuroprotection.

\section{ACKNOWLEDGEMENTS}

This work was supported by the Health Research Council of New Zealand (17/601).

\section{AUTHOR CONTRIBUTIONS}

J.O.G., L.B., S.T., and A.J.G. contributed to the conception and design of the manuscript, drafting the article, and approving the final version.

\section{ADDITIONAL INFORMATION}

Competing interests: The authors declare no competing interests.

Publisher's note Springer Nature remains neutral with regard to jurisdictional claims in published maps and institutional affiliations.

\section{REFERENCES}

1. Gunn, A. J. et al. Therapeutic hypothermia translates from ancient history in to practice. Pediatr. Res. 81, 202-209 (2017).

2. Ferriero, D. M. Neonatal brain injury. N. Engl. J. Med. 351, 1985-1995 (2004).

3. Favié, L.M.A. et al. Pharmacokinetics and short-term safety of the selective NOS inhibitor 2-iminobiotin in asphyxiated neonates treated with therapeutic hypothermia. Pediatr Res. (2019) https://doi.org/10.1038/s41390-019-0587-1 [Epub ahead of print] PMID: 31578035.

4. Bjorkman, S. T. et al. Short-term dose-response characteristics of 2-iminobiotin immediately postinsult in the neonatal piglet after hypoxia-ischemia. Stroke $\mathbf{4 4}$, 809-811 (2013).

5. Favie, L. M. A. et al. Nitric oxide synthase inhibition as a neuroprotective strategy following hypoxic-ischemic encephalopathy: evidence from animal studies. Front. Neurol. 9, 258 (2018).

6. Jacobs, S., Hunt, R., Tarnow-Mordi, W., Inder, T. \& Davis, P. Cooling for newborns with hypoxic-ischaemic encephalopathy. Cochrane Database Syst. Rev. 4, CD003311 (2007).

7. $\mathrm{Wu}, \mathrm{Y}$. W. et al. High-dose erythropoietin and hypothermia for hypoxic-ischemic encephalopathy: a phase II trial. Pediatrics 137, e20160191 (2016).

8. Wassink, G. et al. A working model for hypothermic neuroprotection. J. Physiol. 596, 5641-5654 (2018).

9. Herz, J. et al. Interaction between hypothermia and delayed mesenchymal stem cell therapy in neonatal hypoxic-ischemic brain injury. Brain Behav. Immun. 70, 118-130 (2018).

10. Thoresen, M. et al. Post-hypoxic hypothermia reduces cerebrocortical release of NO and excitotoxins. NeuroReport 8, 3359-3362 (1997).

11. Fujioka, $\mathrm{H}$. et al. Biopterin in the acute phase of hypoxia-ischemia in a neonatal pig model. Brain Dev. 30, 1-6 (2008).

12. $\mathrm{Wu}, \mathrm{J}$. et al. Changes of endothelin-1 and nitric oxide systems in brain tissue during mild hypothermia in a porcine model of cardiac arrest. Neurocrit. Care (2019).

13. Davidson, J. O. et al. How long is too long for cerebral cooling after ischemia in fetal sheep? J. Cereb. Blood Flow Metab. 35, 751-758 (2015).

14. Shankaran, S. et al. Effect of depth and duration of cooling on death or disability at age 18 months among neonates with hypoxic-ischemic encephalopathy: a randomized clinical trial. JAMA 318, 57-67 (2017).

15. Edwards, A. D. et al. Neurological outcomes at 18 months of age after moderate hypothermia for perinatal hypoxic-ischaemic encephalopathy: synthesis and meta-analysis of trial data. BMJ 340, c363 (2010). 